\title{
INTÉGRITÉ GÉNOMIQUE ET REPEUPLEMENTS CHEZ LA TRUITE COMMUNE DU VERSANT MÉDITERRANÉEN.
}

\author{
C. POTEAUX et P. BERREBI
}

Laboratoire Génome et Populations, CNRS - UPR 9060, Case 063,
Place Eugène Bataillon, 34095 Montpellier Cedex 05, France.

\begin{abstract}
RÉSUMÉ
La truite commune est une espèce manipulée depuis près d'un siècle. Pour remplacer l'effectif prélevé par la pêche, des pratiques de déversements de truites d'élevage ont été mises en place très tôt. Pour la truite sauvage du versant méditerranéen, ces repeuplements résultent en une introgression génétique des truites résidantes par les truites d'élevage, génétiquement différenciées. Nous avons effectué dans cet article une revue des données protéiques obtenues pour de nombreux échantillons méditerranéens dans notre laboratoire, en estimant l'introgression domestique et en essayant de décrire les conséquences génétiques des repeuplements sur les populations en place. Quelques solutions de remplacement aux déversements classiques de truites d'élevage sont discutées.
\end{abstract}

Mots-clés : repeuplement, introgression, génétique, Salmo trutta.

\section{GENOME INTEGRITY AND TROUT STOCKING IN THE MEDITERRANEAN BASIN.}

\section{ABSTRACT}

The stocking practices used for brown trout (Salmo trutta) in the South of France result in secondary contact and introgression between populations from two genetically differentiated forms of the same species (KRIEG and GUYOMARD, 1985 ; GUYOMARD, 1989) : domesticated stocks that originate from the Atlantic form and the wild Mediterranean populations.

This paper reviews the protein data for 44 Mediterranean samples examined in the laboratory, with an appraisal of introgression by the domesticated form and a description of the genetic consequences of stocking on the existing populations. The samples were collected from several French departments : Pyrénées Orientales (12 stations), Hérault (5 stations), Vaucluse (2 stations), and Corsica (25 stations) (see Table I). Electrophoresis on horizontal starch gel revealed 28 loci. Introgression was quantified using three diagnostic protein loci : lactate dehydrogenase of the eye $\left(\mathrm{LDH}-5^{*}\right)$, serum transferrin (TF*) and fructose biphosphatase of liver $\left(F B P-1^{*}\right)$. The individuals identified as domesticated fish on the basis of their genotype were discarded. Frequencies at diagnostic loci, expected heterozygosity, the deviation from panmixia in each sample, and domestic introgression are given in Table II. Heterozygote deficit was estimated using parameter $f$ of WEIR and COCKHERAM (1984). Likewise, parameter $\theta$ was used to assess whether the structure of the different samples was statistically significant. The values of these two parameters were tested by permutation using the GENETIX 2.0 programme (BELKHIR et al., submitted). The populations analysed can be classified according to their degree of introgression, which ranged from 0 to $77 \%$. Most introgressed populations displayed substantial and significant heterozygote deficit. Stocking is the most likely cause of these imbalances. However, this interpretation cannot be generalised to all the samples because the deficit did not increase with the severity of introgression. 
The results as a whole tend to show that stocking has a genetic effect on natural populations (with changes in their genotype, and imbalances in panmixia and linkages). The data also illustrate the weak effect of stocking on population densities. This has already been reported by several authors. The various causes for this lack of success in stocking (stocking techniques, learning problems in the wild environment, domestication) are discussed.

Introgression-free Mediterranean populations generally occur at high altitudes and they are practically monomorphic. The advantages of conserving genetically pure populations with no polymorphism are discussed. Two solutions that reconcile objectives as different as supporting trout population densities and protecting biodiversity are examined : the creation of local strains, which requires rigorous genetic management (CHEVASSUS, 1989) and the introduction of triploid individuals, which would result in solely ecological interactions. Finally, the question of the complete interruption of stocking in the whole area or in "genetic sanctuaries" that have been identified as genetically original is discussed.

Other diagnostic markers distinguishing trout of the two origins have been described recently. They provide information on introgression at levels other than that of proteins. The study of the mitochondrial genome gives data on the maternal origin of each individual and hence on introgression through the mother. Finally, the study of highly polymorphic microsatellite markers will make it possible to distinguish between the natural and domesticated components of the populations of the Atlantic basin, a zone in which there are no diagnostic allozymic loci for these two forms.

Key-words : re-stocking, introgression, genetics, Salmo trutta.

\section{INTRODUCTION}

Les pratiques de repeuplement employées dans le sud de la France pour la truite commune (Salmo trutta fario) équivalent à remettre en contact deux formes différenciées de la même espèce : la forme atlantique (issue du versant atlantique et modifiée par la domestication) et la forme méditerranéenne sauvage. Sans être une introduction d'espèce proprement dite, il s'agit bien d'une introduction d'un taxon, suffisamment différencié du taxon récepteur pour créer les déséquilibres décrits plus bas. Ceci constitue d'ailleurs un bon exemple où l'espèce n'est pas le bon niveau d'intégration et où le taxon (ici, c'est le niveau infraspécifique) lui est préférable (SASTRE et BENTATA, 1997).

Des taxons vivant en allopatrie pendant un laps de temps suffisamment long vont se différencier de façon indépendante et certaines discontinuités génétiques peuvent se fixer (DOBZHANSKY, 1950). La remise en contact des génomes différenciés peut se traduire par une absence d'interactions génétiques entre les deux taxons si l'isolement reproducteur est total, ou par une hybridation s'il n'est que partiel. Dans le cas de génomes peu différenciés, l'isolement reproducteur est très limité ou n'existe pas. La remise en contact de deux génomes peut avoir une origine anthropique, par introduction de l'une des deux formes dans une région où l'autre forme est présente. Ainsi, il n'y aura pas de zone de contact proprement dite mais une dilution du pool génique introduit dans le pool du taxon qui le reçoit. Les hybridations seront en général fertiles et la composition génétique des taxons concernés sera susceptible d'être modifiée par des croisements successifs, donnant lieu à ce qu'on appelle une introgression (MAYR, 1974).

C'est le cas de la truite commune en France, pour laquelle les deux formes citées précédemment correspondent à des zones géographiques distinctes (KRIEG et GUYOMARD, 1985 ; PRESA et al., 1994) : la forme méditerranéenne, localisée sur le pourtour nord du bassin méditerranéen, et la forme atlantique qui occupe le reste de l'aire de distribution de cette espèce. Dans le cas de la truite méditerranéenne, l'hybridation est due à un contact secondaire causé par l'introduction dans le milieu, par l'homme, d'une forme génétiquement différenciée : la forme domestique (LETERRIER et al., 1989; BEAUDOU et al., 1993). Les truites d'élevage dérivent toutes de souches d'origine atlantique. Elles ont subi une intense sélection en pisciculture, involontaire ou programmée pour des traits particuliers, ce qui contribue à augmenter plus encore leur différenciation par rapport aux populations naturelles du sud. 
Ces manipulations et les introductions liées à l'homme ont concerné de nombreux pays. Elles ont contribué à augmenter l'aire de répartition naturelle de la truite commune, qui s'étend, latitudinalement, du nord de la Scandinavie aux versants de l'Atlas en Afrique du Nord, et longitudinalement, de l'Islande à la mer d'Aral (BEHNKE, 1972). Son implantation dans des aires nouvelles a en général très bien réussi de l'Amérique du Nord à presque tout l'hémisphère Sud (MACCRIMMON et MARSHALL, 1968 ; GUYOMARD et al., 1984 ; ELLIOTT, 1989). En revanche, on estime que les implantations locales dans des rivières situées sur son aire originelle sont faibles d'après les résultats d'études de marquage-recapture ou d'estimations génétiques. Sur le versant méditerranéen, où cette implantation se traduit par une introgression avec la truite autochtone, ce taux varie de presque $0 \%$ (MORAN et al., 1991 et 1993 ; BEAUDOU, 1993) à plus de $40 \%$ (LETERRIER et al., 1989).

Ces études génétiques reposent sur la technique de l'électrophorèse des protéines enzymatiques, qui est appliquée au niveau populationnel depuis 1966 (BUTH, 1984 ; PASTEUR et al., 1987). L'étude du polymorphisme protéique permet de détecter la variabilité au niveau des locus qui codent pour ces protéines. Chez la truite commune, des études allozymiques importantes ont débuté assez tôt dans les pays anglo-saxons (ALLENDORF et al., 1977 ; ALLENDORF et UTTER, 1979) et nordiques (RYMAN et al., 1979), et ont permis de mettre en évidence une importante structure génétique chez cette espèce. Certains locus se sont montrés particulièrement intéressants pour leur valeur phylogéographique (HAMILTON et al., 1989) et pour leur pouvoir de discrimination entre formes sauvages et stocks d'élevage (revue dans UTTER et SEEB, 1990).

Sur le versant méditerranéen, l'étude des protéines solubles permet de distinguer les formes domestiques d'origine atlantique des truites sauvages méditerranéennes, grâce à deux locus diagnostiques (lactate deshydrogénase de l'oeil, $L D H-5^{*}$ et transferrine du sérum, $T F^{*}$ ). Un troisième locus, la fructose biphosphatase du foie $\left(F B P-1^{*}\right)$, s'est révélé informatif sur le versant méditerranéen car il est fixé chez plusieurs populations non-introgressées (KRIEG et GUYOMARD, 1985 ; LETERRIER et al., 1989 ; PRESA et al., 1994).

Nous nous proposons dans le présent article de faire une revue des données protéiques obtenues pour le versant méditerranéen français dans notre laboratoire. Nous essaierons de dégager les conséquences génétiques des repeuplements sur les populations en place et de donner quelques indications pour une meilleure gestion de cette espèce.

\section{MATÉRIEL ET MÉTHODES}

\section{Echantillonnage}

Les données exposées dans cet article ont été obtenues lors d'études commandées par divers organismes cités dans les remerciements, et lors de diplômes soutenus dans le laboratoire Génome et Populations de l'Université Montpellier II. Dans un souci d'homogénéisation, nous reprenons la liste complète des échantillons analysés dans le tableau I, qui présente également les principales caractéristiques des différentes stations. Ainsi, les Pyrénées Orientales ont été échantillonnées à 13 stations réparties sur 5 bassins hydrographiques. Dans le département de l'Hérault, des prélèvements ont été effectués en plusieurs points des réseaux de l'Orb et de l'Hérault. Pour le Vaucluse, deux points de la Sorgue ont été analysés, près de la source et plus à l'aval. Enfin, 25 stations ont été pêchées en Corse, à la fois sur la côte est et la côte ouest.

Deux échantillons de truites d'élevage ont été analysés également, afin d'avoir une référence domestique. 30 truites ont été prélevées pendant l'été 1993 à la pisciculture de Brassac (Tarn) et à celle de la Canourgue (Lozère). 


\section{Tableau I}

Liste des échantillons analysés.

Table 1

List of analysed samples.

\begin{tabular}{|c|c|c|c|c|c|}
\hline Département & Bassin & Echantillon & Rivière & Effectif & Référence \\
\hline $\begin{array}{l}\text { Pyrénées } \\
\text { Orientales }\end{array}$ & $\begin{array}{l}\text { Sègre } \\
\text { Tech } \\
\text { Tet } \\
\text { Agly } \\
\text { Aude }\end{array}$ & $\begin{array}{c}1 \\
2 \\
3 \\
4 \\
5 \\
6 \\
7 \\
8 \\
9 \\
10 \\
11 \\
12\end{array}$ & $\begin{array}{c}\text { Eyne } \\
\text { Campcardos } \\
\text { amont Tech } \\
\text { Riu Ferrer } \\
\text { Carança } \\
\text { Alemany } \\
\text { Campeille } \\
\text { Nohèdes } \\
\text { amont } \\
\text { Lladure } \\
\text { Boutadiol } \\
\text { Aude amont }\end{array}$ & $\begin{array}{c}24 \\
23 \\
18 \\
27 \\
28 \\
11 \\
8 \\
16 \\
13 \\
21 \\
28 \\
30\end{array}$ & Berrebi (1995b) \\
\hline Hérault & $\begin{array}{c}\text { Hérault } \\
\text { Orb }\end{array}$ & $\begin{array}{l}13 \\
14 \\
15 \\
16 \\
17\end{array}$ & $\begin{array}{c}\text { Laurounet } \\
\text { Brèze } \\
\text { Vis } \\
\text { Orb } \\
\text { Tes }\end{array}$ & $\begin{array}{l}18 \\
10 \\
20 \\
113 \\
110\end{array}$ & $\begin{array}{l}\text { Berrebi (1995d) } \\
\text { Poteaux (1995) }\end{array}$ \\
\hline Vaucluse & Sorgue & $\begin{array}{l}18 \\
19\end{array}$ & $\begin{array}{c}\text { amont } \\
\text { aval }\end{array}$ & $\begin{array}{l}30 \\
21\end{array}$ & $\begin{array}{l}\text { Poteaux (1995) } \\
\text { Berrebi (1995a) }\end{array}$ \\
\hline Corse & $\begin{array}{c}\text { Tavignano } \\
\text { Golo } \\
\text { Porto } \\
\text { Fium Alto } \\
\text { Prunelli } \\
\text { Liamone } \\
\text { Prunelli } \\
\\
\\
\text { Taravo } \\
\\
\text { Fium'Orbo } \\
\text { Abatesco } \\
\text { Travo } \\
\text { Golo } \\
\text { Taravo } \\
\text { Fium'Orbo } \\
\text { Rizzanese } \\
\text { Solenzara } \\
\text { Golo } \\
\text { Bravone } \\
\text { Furcone }\end{array}$ & $\begin{array}{l}20 \\
21 \\
22 \\
23 \\
24 \\
25 \\
26 \\
27 \\
28 \\
29 \\
30 \\
31 \\
32 \\
33 \\
34 \\
35 \\
36 \\
37 \\
38 \\
39 \\
40 \\
41 \\
42 \\
43 \\
44\end{array}$ & $\begin{array}{c}\text { amont } \\
\text { Verghello } \\
\text { Golo aval } \\
\text { Golo amont } \\
\text { Tavulella } \\
\text { Fium Alto } \\
\text { Gravone } \\
\text { Cruzini } \\
\text { Liamone } \\
\text { amont } \\
\text { Calderamolla } \\
\text { Montichi } \\
\text { Taravo amont } \\
\text { Veraculungo } \\
\text { Marmano } \\
\text { Abatesco } \\
\text { Travo } \\
\text { Melaja } \\
\text { Chiova } \\
\text { Pozzi } \\
\text { San Petru } \\
\text { Renaju } \\
\text { Casaluna } \\
\text { amont } \\
\text { Furcone }\end{array}$ & $\begin{array}{l}27 \\
18 \\
30 \\
27 \\
35 \\
29 \\
31 \\
31 \\
30 \\
31 \\
34 \\
22 \\
30 \\
28 \\
29 \\
32 \\
28 \\
17 \\
30 \\
25 \\
28 \\
30 \\
16 \\
30 \\
23\end{array}$ & Berrebi (1995c) \\
\hline Pisciculture & $\begin{array}{l}\text { Lozère } \\
\text { Tarn }\end{array}$ & $\begin{array}{l}45 \\
46\end{array}$ & $\begin{array}{c}\text { Canourgue } \\
\text { Brassac }\end{array}$ & $\begin{array}{l}30 \\
30\end{array}$ & Poteaux (1995) \\
\hline
\end{tabular}




\section{Méthodes}

Lors de chaque campagne, les poissons ont été disséqués sur place et les organes utilisés pour l'étude allozymique (yeux, sang, foie et muscle) ont été prélevés et congelés instantanément dans de l'azote liquide pour leur conservation jusqu'à l'analyse.

Les électrophorèses ont été faites sur gel horizontal d'amidon à 11,4\%. La composition des tampons de migration et les conditions de révélation sont tirées de PASTEUR et al. (1987) ou de BEAUDOU (1993). La nomenclature des locus est celle de SHAKLEE et al. (1990). L'analyse a porté sur quatorze systèmes enzymatiques qui ont permis la révélation de 28 locus dont le déterminisme génétique a été vérifié pour la plupart (GUYOMARD et KRIEG, 1983; BARBAT-LETERRIER et al., 1989).

Nous ne préciserons dans les résultats que les fréquences aux locus diagnostiques. Par contre, l'hétérozygotie théorique est basée sur la totalité des 28 locus analysés.

Les échantillons ayant été prélevés à différentes époques de l'année, plus ou moins proches de périodes de déversements intenses, nous avons éliminé des analyses les individus identifiés d'après leur génotype comme pouvant être originaires de pisciculture.

Le paramètre $f$ de WEIR et COCKERHAM (1984) est utilisé pour estimer la valeur du déficit d'hétérozygotes et le paramètre $\theta$ est un estimateur de la structuration entre les différents échantillons. Les calculs de ces deux paramètres sont basés sur les génotypes aux 28 locus enzymatiques analysés. La valeur obtenue pour ces deux paramètres a été testée par permutation, avec le logiciel GENETIX 2.0 (BELKHIR et al., soumis).

Une estimation de l'introgression est calculée à partir des fréquences obtenues aux trois seuls locus diagnostiques. Ce calcul se fait sous l'hypothèse d'une fixation des allèles méditerranéens dans les peuplements purement méditerranéens (comme déjà observé à plusieurs stations Carança, Vis, Golo). Nous estimons donc que tout allèle atlantique est d'origine exogène. Le taux d'introgression est la moyenne arithmétique des pourcentages de gènes atlantiques relevés à ces trois locus. Notons toutefois que la présence (faible) d'un allèle " méditerranéen " au locus FBP-1" dans la plupart des souches de pisciculture provoque une légère sous-estimation de ce taux.

\section{RÉSULTATS}

Les fréquences alléliques aux locus diagnostiques sont présentées pour l'ensemble des échantillons dans le tableau II. Dans ce tableau sont indiquées également la valeur de l'hétérozygotie attendue $(\mathrm{He})$ et la valeur du test de déficits d'hétérozygotes (f), ces deux paramètres étant calculés sur les 28 locus enzymatiques.

Les différences génétiques par paires d'échantillons (paramètre $\theta$ ) sont indiquées dans le tableau III. Ce tableau nous montre que les échantillons sont fortement structurés en fonction de leur niveau d'introgression. Trois ensembles significativement différents sont représentés par 1) les populations pas ou peu introgressées, par exemple l'Aude dans les Pyrénées et Tavignano en Corse, 2) celles qui le sont très sensiblement (environ $30 \%$ dans Boutadiol, dans les Pyrénées et Tavulella en Corse) et 3) les populations très introgressées, dont les caractéristiques génétiques se rapprochent plus de celles de pisciculture que des naturelles (Nohèdes dans les Pyrénées et Melaja en Corse, par exemple).

Les valeurs d'introgression calculées à partir des trois locus diagnostiques sont présentées au tableau II. Ces valeurs varient de $0 \%$ pour les échantillons purs méditerranéens (ex. : Carença, Vis, Golo amont ou Marmano) à plus de $75 \%$ de gènes domestiques (Melaja ou Soulondre). Certaines populations ne sont constituées que d'individus domestiques (ex. : Agly) mais elles constituent probablement des populations " temporaires ", maintenues en place uniquement par la volonté de l'homme. 


\section{Tableau II}

Fréquences alléliques aux trois locus diagnostiques, hétérozygotie (He), déséquilibre d'hétérozygotes ( $f$ ) et taux d'introgression (I en \%). Pyrénées Orientales (a) et Corse (b). La station Agly ne figure pas au tableau, car elle est entièrement composée de truites domestiques.

$$
{ }^{\star}=P<0,05 ;{ }^{\star \star}=P<0,01 ;{ }^{\star \star \star}=P<0,001 \text {. }
$$

\section{Table II}

Allelic frequencies at the three diagnostic loci, heterozygosity (He), hétérozygotes disequilibrium (f) and introgression index ( $\mathrm{l}$ in \%) in Pyrénées Orientales (a) and Corsica (b). Data of Agly sample are not given because composed of domestic trout only.

$$
{ }^{\star}=P<0.05 ;{ }^{\star \star}=P<0.01 ;{ }^{\star \star \star}=P<0.001 .
$$

\begin{tabular}{|c|c|c|c|c|c|c|c|c|c|c|c|c|c|c|c|c|c|c|}
\hline & \multicolumn{11}{|c|}{ Pyrénées Orientales } & \multicolumn{5}{|c|}{ Hérault } & \multicolumn{2}{|c|}{ Vaucluse } \\
\hline & $i$ & 2 & 3 & 4 & 5 & 6 & 7 & 8 & 10 & 11 & 12 & 13 & 14 & 15 & 16 & 17 & 18 & 19 \\
\hline FBP1 & & & & & & & & & & & & & & & & & & \\
\hline & 21 & 12 & 18 & 25 & 28 & 10 & 6 & 16 & 17 & 28 & 27 & 18 & 10 & 19 & 113 & 110 & 30 & 21 \\
\hline 100 & 0,14 & 0,42 & 0,39 & 0,06 & - & 0.45 & - & 0,75 & 0,24 & 0,29 & 0.02 & 0.5 & 0,2 & 0,05 & 0,13 & 0,07 & 0.03 & 0,18 \\
\hline 150 & 0.86 & 0.58 & 0.61 & 0,94 & 1 & 0.55 & 1 & 0,25 & 0.77 & 0.71 & 0.98 & 0,5 & 0,8 & 0.95 & 0.87 & 0,93 & 0,97 & 0,82 \\
\hline \multicolumn{19}{|l|}{ LDH5 } \\
\hline$N$ & 22 & 22 & 18 & 26 & 28 & 11 & 8 & 16 & 21 & 28 & 28 & 18 & 10 & 19 & 104 & 85 & 30 & 21 \\
\hline 90 & 0.18 & 0.23 & 0,36 & 0.23 & - & 0,46 & - & 0.59 & 0,12 & 0.14 & 0.02 & 0.31 & 0,05 & . & 0.18 & 0.08 & . & 0.09 \\
\hline 100 & 0.82 & 0.77 & 0,58 & 0,77 & 1 & 0,55 & 1 & 0,41 & 0.86 & 0.86 & 0,98 & 0.69 & 0,95 & 1 & 0.82 & 0.92 & 1 & 0.91 \\
\hline 110 & . & - & 0,06 & - & 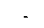 & - & - & - & 0,02 & 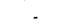 & & & - & . & 0,01 & . & - & - \\
\hline \multicolumn{19}{|l|}{ TF1 } \\
\hline & 21 & 19 & 16 & 27 & 28 & 11 & 8 & 16 & 20 & 19 & 28 & - & - & . & 66 & 73 & 30 & 21 \\
\hline 100 & 0,19 & 0,58 & 0,25 & 0,28 & - & 0,5 & - & 0,97 & 0,35 & 0,4 & - & . & . & - & 0,38 & 0,15 & - & 0.27 \\
\hline 102 & 0,81 & 0,42 & 0,75 & 0,72 & 1 & 0.5 & 1 & 0,03 & 0,65 & 0,61 & 1 & - & - & - & 0.62 & 0.85 & 1 & 0.73 \\
\hline $\mathrm{He}(\%)$ & 5,97 & 7,9 & 8,4 & 4,99 & - & 12.4 & 1,1 & 10,8 & 5,8 & 7,3 & 1.35 & 8,3 & 4,5 & 2,3 & 7,66 & 5,32 & 0,7 & 5,95 \\
\hline $\begin{array}{l}t \\
P\end{array}$ & 0.29 & 0.36 & 0,42 & 0.22 & - & 0.41 & -0.17 & 0,05 & 0,48 & $\begin{array}{l}0.67 \\
\therefore .\end{array}$ & 0,31 & 0.05 & 0,02 & 0,54 & 0,11 & 0.06 & - & $\begin{array}{l}0,37 \\
\therefore \cdots\end{array}$ \\
\hline $\mid(\%)$ & 17 & 41 & 33 & 19 & 0 & 47 & 0 & 77 & 24 & 28 & 1 & 40 & 10 & 2 & 24 & 11 & 1 & 18 \\
\hline
\end{tabular}

\begin{tabular}{|c|c|c|c|c|c|c|c|c|c|c|c|c|c|c|c|c|c|c|c|c|c|c|c|c|c|}
\hline \multicolumn{26}{|c|}{ Corse } \\
\hline & 20 & 21 & 22 & 23 & 24 & 25 & 26 & 27 & 28 & 29 & 30 & $\overline{31}$ & 32 & 33 & 34 & 35 & 36 & 37 & 38 & 39 & 40 & 41 & 42 & 43 & 44 \\
\hline $\begin{array}{c}\text { FBP } 1 \\
N \\
100 \\
150\end{array}$ & $\begin{array}{c}23 \\
- \\
1\end{array}$ & $\begin{array}{l}15 \\
0.1 \\
0.9\end{array}$ & $\begin{array}{c}28 \\
1\end{array}$ & $\begin{array}{c}10 \\
i \\
1\end{array}$ & $\begin{array}{l}21 \\
0,1 \\
0,9\end{array}$ & $\begin{array}{l}17 \\
0.8 \\
0.2\end{array}$ & $\begin{array}{c}30 \\
- \\
1\end{array}$ & $\begin{array}{l}31 \\
;\end{array}$ & $\begin{array}{c}30 \\
1\end{array}$ & $\begin{array}{l}31 \\
0.7 \\
0.3\end{array}$ & $\begin{array}{r}34 \\
- \\
1\end{array}$ & $\begin{array}{l}22 \\
0.3 \\
0.7\end{array}$ & $\begin{array}{c}30 \\
0 \\
1\end{array}$ & $\begin{array}{c}28 \\
-1\end{array}$ & $\begin{array}{c}29 \\
1\end{array}$ & $\begin{array}{c}32 \\
0 \\
1\end{array}$ & $\begin{array}{l}27 \\
0.3 \\
0.7\end{array}$ & $\begin{array}{l}14 \\
0.7 \\
0.3\end{array}$ & $\begin{array}{c}30 \\
i \\
i\end{array}$ & $\begin{array}{c}25 \\
i\end{array}$ & $\begin{array}{l}26 \\
0.3 \\
0.7\end{array}$ & $\begin{array}{l}30 \\
0.4 \\
0.6\end{array}$ & $\begin{array}{c}16 \\
\vdots \\
1\end{array}$ & $\begin{array}{l}30 \\
0.2 \\
0.8\end{array}$ & $\begin{array}{l}2.3 \\
0.3 \\
0.7\end{array}$ \\
\hline $\begin{array}{l}\text { LOH5 } \\
90 \\
90 \\
100 \\
110\end{array}$ & $\begin{array}{c}27 \\
0 \\
1\end{array}$ & $\begin{array}{l}18 \\
0,1 \\
0.9\end{array}$ & $\begin{array}{c}29 \\
0 \\
1\end{array}$ & $\begin{array}{c}27 \\
1\end{array}$ & $\begin{array}{l}34 \\
0,4 \\
0,6\end{array}$ & $\begin{array}{l}26 \\
0,2 \\
0,8\end{array}$ & $\begin{array}{l}31 \\
0.1 \\
0.9\end{array}$ & $\begin{array}{r}31 \\
1\end{array}$ & $\begin{array}{c}30 \\
0 \\
1\end{array}$ & $\begin{array}{l}30 \\
0.3 \\
0.7\end{array}$ & $\begin{array}{c}34 \\
- \\
1\end{array}$ & $\begin{array}{l}22 \\
0.3 \\
0.8\end{array}$ & $\begin{array}{l}30 \\
0.2 \\
0.8\end{array}$ & $\begin{array}{c}28 \\
i\end{array}$ & $\begin{array}{c}29 \\
i\end{array}$ & $\begin{array}{l}32 \\
0.1 \\
0.9\end{array}$ & $\begin{array}{c}28 \\
0 \\
1\end{array}$ & $\begin{array}{l}17 \\
0.7 \\
0,4\end{array}$ & $\begin{array}{c}30 \\
0 \\
1\end{array}$ & $\begin{array}{c}25 \\
i\end{array}$ & $\begin{array}{l}28 \\
0.5 \\
0.5\end{array}$ & $\begin{array}{c}30 \\
0 \\
1\end{array}$ & $\begin{array}{c}16 \\
- \\
1\end{array}$ & $\begin{array}{l}30 \\
0.6 \\
0.5\end{array}$ & $\begin{array}{l}23 \\
0.3 \\
0.7\end{array}$ \\
\hline $\begin{array}{l}\text { TF1 } \\
\text { N } \\
100 \\
102 \\
80 \\
70\end{array}$ & $\begin{array}{c}27 \\
0 \\
1 \\
-\end{array}$ & $\begin{array}{c}15 \\
0,1 \\
0.9 \\
- \\
-\end{array}$ & $\begin{array}{c}30 \\
- \\
1 \\
-\end{array}$ & $\begin{array}{c}20 \\
\dot{1} \\
- \\
-\end{array}$ & $\begin{array}{c}28 \\
0.3 \\
0.7 \\
.\end{array}$ & $\begin{array}{c}18 \\
0,2 \\
0.8 \\
- \\
-\end{array}$ & $\begin{array}{c}25 \\
- \\
1 \\
-\end{array}$ & $\begin{array}{c}26 \\
0 \\
1 \\
-\end{array}$ & $\begin{array}{c}28 \\
0.1 \\
0.9 \\
0\end{array}$ & $\begin{array}{l}37 \\
0.1 \\
0.5 \\
0.4 \\
0\end{array}$ & $\begin{array}{r}29 \\
- \\
1 \\
-\end{array}$ & $\begin{array}{l}22 \\
0.4 \\
0.6\end{array}$ & $\begin{array}{l}21 \\
0,3 \\
0,5 \\
0,1\end{array}$ & $\begin{array}{c}28 \\
1 \\
- \\
-\end{array}$ & $\begin{array}{c}29 \\
1 \\
- \\
-\end{array}$ & $\begin{array}{c}30 \\
0,1 \\
1 \\
-\end{array}$ & $\begin{array}{c}27 \\
\vdots \\
1 \\
-\end{array}$ & $\begin{array}{c}15 \\
0.7 \\
0.3 \\
- \\
-\end{array}$ & $\begin{array}{l}29 \\
0,2 \\
0,1 \\
0,7\end{array}$ & $\begin{array}{l}24 \\
0.1 \\
0.9\end{array}$ & $\begin{array}{c}15 \\
0.5 \\
0.5 \\
.\end{array}$ & $\begin{array}{c}29 \\
0 \\
1 \\
-\end{array}$ & $\begin{array}{c}15 \\
0.2 \\
0.8 \\
-\end{array}$ & $\begin{array}{c}27 \\
0.6 \\
0.4 \\
:\end{array}$ & $\begin{array}{c}23 \\
0.2 \\
0.8 \\
- \\
-\end{array}$ \\
\hline $\mathrm{He}(\%)$ & 2,6 & 4,5 & 1.5 & 0,4 & 7,5 & 6,4 & 2,3 & 3.2 & 4,3 & 9,4 & - & 12 & 6,8 & - & 0.8 & 4.2 & 3.9 & 8.3 & 5,1 & 1 & 12 & 5 & 3.8 & 10 & 8.9 \\
\hline i & 0 & 0.4 & .0 .1 & .0 & -0.2 & $0: 1$ & 0.1 & $-0,1$ & .0 .2 & 0 & - & $\begin{array}{l}0,2 \\
-2\end{array}$ & 0 & . & 0,3 & 0,1 & 0,1 & $-0,1$ & .0 & 0.6 & 0 & 0.1 & 0,7 & -0.1 & -0.1 \\
\hline $\mid(\%)$ & 2 & 9 & 1 & 0 & 29 & 39 & 3 & 1 & 2 & 36 & 0 & 32 & 18 & 0 & 0 & 5 & 10 & 69 & 6 & 3 & 45 & 14 & 7 & 44 & 26 \\
\hline
\end{tabular}

\section{DISCUSSION}

Les allèles atlantiques sont présents dans nos échantillons méditerranéens repeuplés en fréquence très variable : on observe peu d'introgressions effectives dans certaines stations ( 0 à $5 \%$ d'allèles domestiques) jusqu'à des valeurs assez élevées pour d'autres (77\% pour une station des Pyrénées Orientales). On observe notamment un impact direct des repeuplements chez certaines populations échantillonnées plusieurs années (l'Orb par exemple) pour lesquelles on n'a pas de stabilité des fréquences alléliques dans le temps (par exemple : $11 \%$ en 1991 et $24 \%$ en 1993 dans l'Orb, POTEAUX, 1995). 
Tableau III

Matrice des différences par paires d'échantillons pour les Pyrénées Orientales (a) et la Corse (b).

Table III

Matrix of genetic distances between samples of Pyrénées Orientales (a) and Corsica (b).

a -

\begin{tabular}{|c|c|c|c|c|c|c|c|c|c|c|}
\hline 3 & 4 & 6 & 7 & 10 & 2 & 11 & 5 & 8 & 1 & 12 \\
\hline Tech & 0.06 & 0.02 & 0.14 & 0.02 & 0.02 & 0.02 & 0.31 & 0.21 & 0.04 & 0.24 \\
\hline Riuferrer & - & 0.14 & 0.105 & 0.02 & 0.11 & 0.07 & 0.18 & 0.39 & 0.02 & 0.14 \\
\hline Alemany & & - & 0.24 & 0.11 & 0.02 & 0.10 & 0.48 & 0.09 & 0.12 & 0.42 \\
\hline Campeille & & & - & 0.10 & 0.21 & 0.14 & 0.32 & 0.48 & 0.06 & 0.07 \\
\hline Lladure & & & & - & 0.03 & 0.015 & 0.22 & 0.33 & 0.03 & 0.17 \\
\hline Campcardos & & & & & - & 0.02 & 0.39 & 0.162 & 0.09 & 0.33 \\
\hline Boutadiol & & & & & & - & 0.25 & 0.275 & 0.07 & 0.21 \\
\hline Caranca & & & & & & & - & 0.66 & 0.15 & 0.03 \\
\hline Nohèdes & & & & & & & & - & 0.36 & 0.62 \\
\hline Eyne & & & & & & & & & - & 0.12 \\
\hline Aude & & & & & & & & & & - \\
\hline
\end{tabular}

b -

\begin{tabular}{|c|c|c|c|c|c|c|c|c|c|c|c|c|c|c|c|c|c|c|c|c|c|c|c|c|}
\hline 20 & 21 & 22 & 23 & 24 & 25 & 26 & 27 & 28 & 29 & 30 & 31 & 32 & 33 & 34 & 35 & 36 & 37 & 38 & 39 & 40 & 41 & 42 & 43 & 44 \\
\hline Tavignan & 0 & 002 & 008 & 0.23 & 041 & 032 & 0.32 & 016 & 031 & 0.79 & 0.18 & 0125 & 0.53 & 0.49 & 0.24 & 0.21 & 0.47 & 0.487 & 0.72 & 0.26 & 0.11 & 0.26 & 0.30 & 0.11 \\
\hline Verghetlo & & 005 & 011 & 0.18 & 0.27 & 0.22 & 0.24 & 0.10 & 0.19 & 0.74 & 0.09 & 0.07 & 0.45 & 0.39 & 0.15 & 0.10 & 0.35 & 0.397 & 0.61 & 0.17 & 0.02 & 0.14 & 0.23 & 0.03 \\
\hline Golo aval & & - & 001 & 0.24 & 047 & 0.44 & 0.44 & 0.29 & 0.33 & 0.88 & 0.21 & 0.16 & 0.65 & 0.63 & 0.30 & 0.30 & 0.51 & 0.547 & 0.81 & 0.29 & 0.14 & 0.38 & 0.31 & 0.12 \\
\hline Golo amont & & & . & 0.24 & 0.50 & 0.58 & 0.55 & 0.38 & 0.37 & 0.95 & 0.24 & 0.21 & 0.75 & 078 & 0.43 & 037 & 0.53 & 0.62 & 0.88 & 0.31 & 019 & 0.51 & 0.33 & 0.16 \\
\hline Tavulella & & & & . & 0.37 & 0.44 & 0.45 & 0.37 & 0.22 & 0.68 & 009 & 0.14 & 0.55 & 0.53 & 0.36 & 0.35 & 0.19 & 0.44 & 0.62 & 0.08 & 0.22 & 0.34 & 0.04 & 0.05 \\
\hline Fium Aito & & & & & - & 0.36 & 0.35 & 0.32 & 011 & 064 & 0.14 & 0.24 & 0.40 & 0.43 & 0.275 & 0.18 & 0.27 & 0.40 & 0.16 & 0.19 & 0.18 & 0.28 & 0.31 & 0.19 \\
\hline Gravone & & & & & & & 0.05 & 0.09 & 0.35 & 078 & 0.25 & 0.20 & 0.35 & 0.13 & 0.005 & 0.09 & 0.57 & 0.40 & 0.69 & 0.35 & 0.235 & 0.05 & 0435 & 0.26 \\
\hline Cruzinı & & & & & & & . & 0.08 & 0.36 & 069 & 0.26 & 022 & 0.28 & 0.05 & 0.03 & 0.10 & 0.555 & 0.35 & 0.62 & 0.355 & 0.26 & 0.04 & 0.44 & 0.28 \\
\hline Liamone & & & & & & & & & 0.30 & 0.63 & 0.20 & 014 & 0.33 & 0.23 & 0075 & 0.10 & 0.48 & 033 & 0.59 & 0.295 & 0.17 & 008 & 0.37 & 0.20 \\
\hline Prunelli & & & & & & & & & . & 0.48 & 010 & 017 & 0.46 & 0.43 & 0.29 & 0.22 & 0.16 & 0.29 & 0.31 & 012 & 0.14 & 0.28 & 0.20 & 0.11 \\
\hline Cabderamolla & & & & & & & & & & . & 049 & 049 & 0845 & 0.91 & 062 & 0.68 & 0.72 & 0.14 & 0.94 & 053 & 0.67 & 0.73 & 060 & 0.60 \\
\hline Montichi & & & & & & & & & & & . & 005 & 032 & 0.32 & 018 & 0.17 & 0.13 & 026 & 0.40 & 0.01 & 0.11 & 0146 & 006 & 003 \\
\hline Taravo & & & & & & & & & & & & (1) & 0.34 & 0.31 & 015 & 0.16 & 0.25 & 0.23 & 0.51 & 011 & 0.13 & 010 & 096 & 004 \\
\hline Veraculungo & & & & & & & & & & & & & . & 0.4 & 0.22 & 0.30 & 0.63 & 0.49 & 0.76 & 0.415 & 0.41 & 0.33 & 0.51 & 041 \\
\hline Marmano & & & & & & & & & & & & & & - & 0.06 & 0.18 & 0.64 & 0.46 & 0.80 & 042 & 0.35 & 0.14 & 050 & 0.37 \\
\hline Abatesco & & & & & & & & & & & & & & & - & 004 & 0.48 & 032 & 0.55 & 0.28 & 0.16 & 0.01 & 0.37 & 0.19 \\
\hline Travo & & & & & & & & & & & & & & & & - & 0.44 & 0.37 & 045 & 0.27 & 0.07 & 0.08 & 036 & 0.16 \\
\hline Melaja & & & & & & & & & & & & & & & & & 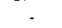 & 049 & 056 & 0.07 & 0.34 & 0.45 & 0.10 & 0.17 \\
\hline Chiova & & & & & & & & & & & & & & & & & & 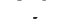 & 0.65 & 0.32 & 0.40 & 0.27 & $0.40^{\circ}$ & 0.34 \\
\hline Pozz: & & & & & & & & & & & & & & & & & & & - & 0.44 & 044 & 0.65 & 0.55 & 048 \\
\hline San Perru & & & & & & & & & & & & & & & & & & & & - & 019 & 0.24 & 0.02 & 0.06 \\
\hline Renaju & & & & & & & & & & & & & & & & & & & & & . & 0.19 & 0.27 & 006 \\
\hline Casalyna & & & & & & & & & & & & & & & & & & & & & & & 033 & 0.17 \\
\hline Bravone & & & & & & & & & & & & & & & & & & & & & & & & 009 \\
\hline Furcone & & & & & & & & & & & & & & & & & & & & & & & & \\
\hline
\end{tabular}

Le niveau de variabilité génétique d'un échantillon dépend de la fréquence des différents allèles présents à chaque locus. Ce niveau de variabilité est peu élevé pour les populations méditerranéennes pures : moins de $2 \%$ pour les populations d'altitude de petite taille (Carança), près de $5 \%$ en Corse, ce qui est probablement plus représentatif des populations méditerranéennes moyennes. Mais la variabilité peut atteindre plus de $11 \%$ pour une population très introgressée (Nohède par exemple), soit une valeur proche de celles rencontrées habituellement chez les populations atlantiques et domestiques (KRIEG et GUYOMARD, 1985). L'introgression domestique résulte donc du passage de gènes atlantiques dans les populations méditerranéennes sauvages, introgression qui s'accompagne d'une augmentation du polymorphisme de la population réceptrice.

La majorité des populations introgressées présentent des déséquilibres (déficits d'hétérozygotes) élevés et significatifs. L'éventualité qu'un effet Wahlund (mélange d'individus issus de deux origines distinctes) soit responsable des déficits observés est à exclure car les individus identifiés d'après leurs génotypes comme originaires de pisciculture ont été éliminés des analyses. Quelle explication peut-on proposer ? II faut remarquer que la valeur de ce déficit n'est pas d'autant plus élevée que l'introgression est forte. En effet, certaines stations 
présentent des déficits élevés et des valeurs d'introgression très faibles, voire nulles (ex. l'Aude, Marmano et Tavignano). D'autre part, de tels déficits ont également été observés chez des populations exemptes de toute introgression : en Corse, la population de Marmano (tableau II) ou en dehors de la région méditerranéenne, dans le Pays Basque (AURELLE, 1996). II y a donc une certaine composante naturelle à ce déséquilibre, mais les perturbations dues aux repeuplements restent la cause la plus probable.

Dans le cas particulier de la Corse, la détection de déséquilibres peut s'expliquer par la présence de deux formes de truites méditerranéennes génétiquement différenciées (KRIEG et GUYOMARD, 1983). Ces truites de «type corse " semblent localisées dans les régions d'altitude de la moitié sud de l'île, et sont mélangées en proportions variables suivant les stations avec des truites méditerranéennes typiques et des individus de repeuplement (BERREBI 1995 c). Cette hypothèse n'est pas applicable à la population de Marmano (97\% de type "corse ancestral "). En revanche, on observe des échantillons présentant des valeurs d'introgression très élevées mais des déficits d'hétérozygotes relativement faibles. C'est le cas pour Nohède, dans les Pyrénées Orientales. Pour cette station, les gènes de pisciculture sont présents à plus de $65 \%$ alors que le déficit ne dépasse pas 0.05 (non significatif). Ceci semble la marque d'introgressions anciennes.

L'introduction répétée des gènes domestiques crée à long terme une perturbation durable de la structure génétique de la population sauvage puisque, outre les déséquilibres en hétérozygotes, des déséquilibres de liaison ont été observés dans certains des échantillons analysés (POTEAUX, 1995). Bien qu'une partie de ces déséquilibres soit éliminée à chaque génération, la présence de ces associations entre allèles de différents locus va perdurer assez longtemps.

Récemment, d'autres marqueurs diagnostiques entre les truites de ces deux origines ont été mis en évidence, par l'étude du génome mitochondrial (par séquençage direct, BERNATCHEZ et al., 1992) et plus récemment encore, grâce aux marqueurs microsatellites (ESTOUP et al., 1993 ; POTEAUX, 1995). Ces deux types de marqueurs apportent des informations sur l'introgression à d'autres niveaux que les protéines. L'ADN mitochondrial renseigne, chez les $\mathrm{F} 1$, sur l'origine maternelle de l'individu uniquement. II nous est donc possible de suivre dans les populations sauvages l'introgression mitochondriale par la voie maternelle et de la comparer à l'introgression nucléaire, transmise par les deux parents. Nous avons pu montrer pour les populations du réseau de l'Orb que l'introgression mitochondriale était plus élevée que l'introgression indiquée par les allozymes (POTEAUX, 1995). Ce résultat peut être en partie imputé à une contribution à la reproduction des femelles domestiques supérieure à celle des mâles d'élevage. Cette différence peut être causée par la compétition sur les frayères entre les mâles sauvages et domestiques, tournant au désavantage de ces derniers.

De même, une étude microsatellitaire a montré, sur les mêmes individus, que l'introgression calculée par les locus microsatellites était beaucoup plus élevée que celle indiquée par les allozymes (POTEAUX et al., soumis). Cette différence peut être attribuée soit à l'indice d'introgression employé pour les microsatellites (“ index maximal d'introgression ") qui diffère de l'indice employé pour les allozymes, soit à une introgression différentielle des deux marqueurs. En effet, les microsatellites sont considérés comme des marqueurs neutres vis-à-vis de la sélection, alors que les marqueurs allozymiques subiraient une contresélection des allèles domestiques après introgression (POTEAUX, 1995). II en découlerait que les allèles des microsatellites se maintiendraient beaucoup mieux et s'accumuleraient. La fréquence de ces allèles peut être d'autant plus forte que les repeuplements sont anciens.

L'ensemble de nos résultats tend à montrer que les repeuplements ont un impact génétique à long terme sur les populations naturelles. Les introductions de formes d'élevage étaient justifiées pour le maintien ou la réhabilitation de l'espèce, menacée par la surexploitation, la destruction (rare) ou la modification de son habitat (pollution, barrage). La littérature nous enseigne que ces introductions ont entraîné, du point de vue génétique, une 
diminution globale de la variabilité des populations naturelles, par élimination des populations natives (par déplacement ou mortalité due à des maladies introduites en même temps que les poissons), ou introgression (HINDAR et al., 1991 ; WAPLES, 1991 ; RYMAN et al., 1993 ; nos données). L'introgression représente à long terme une perte de la ressource génétique adaptée localement par homogénéisation des pools géniques ou rupture des complexes de gènes co-adaptés (LEARY et al., 1995). L'arrêt des introductions ne pourra pas restaurer les formes naturelles présentes avant perturbation dans toute leur diversité. La prise de conscience récente des problèmes posés par les repeuplements rend nécessaire l'identification des stocks pour leur conservation.

Néanmoins, la plupart des études concernant les repeuplements ont reconnu ces pratiques comme étant un échec quant à l'efficacité de l'implantation en terme d'augmentation de densité, quelle que soit l'espèce (ALTUKHOV, 1981 ; GARCIA DE LEANIZ et al., 1989 ; GUYOMARD, 1989 b; BEAUDOU, 1993). Nos données confirment cette impression.

L'explication la plus simple à ce peu de réussite des repeuplements est liée aux techniques de repeuplement elles-mêmes, qui ne facilitent pas toujours la survie des poissons introduits dans le milieu (introduction de centaines d'alevins ou truitelles au même endroit, pas de choix de sites favorables, truites déversées depuis un hélicoptère dans certains lacs d'altitude). Toutefois, un minimum de précautions étant pris en général, l'explication la plus couramment proposée est une mauvaise adaptation des formes d'élevage dans le milieu naturel, du fait de problèmes d'apprentissage et de domestication (FRASER, 1981 ; HINDAR et al., 1991 ; revue dans POTEAUX, 1992). Les conditions d'élevage en pisciculture ne permettent pas un apprentissage des conditions naturelles (territoire de chasse, recherche de nourriture naturelle, courant, comportement face aux prédateurs...) des jeunes stades qui vont être déversés. Pour les souches d'élevage, une sélection intentionnelle est effectuée en pisciculture sur des traits particuliers tels que croissance rapide, résistance aux maladies, fécondité... De nombreux auteurs ont montré que ces manipulations pouvaient entraîner une diminution de la capacité à survivre et à se reproduire dans le milieu naturel (ALLENDORF et UTTER, 1979; ALLENDORF et al., 1987).

Quelques populations naturelles pures ont été observées dans les trois zones étudiées (Carança, Vis et deux stations en Corse). Ces populations sont généralement localisées en altitude (accès difficile d'où intérêt halieutique limité), et quasi-monomorphes. On peut s'interroger sur l'intérêt de conserver des populations certes génétiquement pures mais sans aucun polymorphisme. Les repeuplements entraînent une augmentation de la diversité génique locale et la notion de "pureté génétique " est alors à opposer au caractère d'adaptabilité de cette population à tout changement de son environnement. II est généralement reconnu que le polymorphisme génétique (mesuré ici par les locus allozymiques représentatifs de l'ensemble du génome) est une garantie, pour la population, d'une bonne adaptabilité à tout changement aléatoire de son environnement. Protéger les populations reconnues comme pures, mais toutes très faiblement polymorphes et donc fragiles, ne peut suffire à protéger durablement la forme locale.

Deux objectifs aussi divergents que le soutien de la densité des peuplements de truites et la protection de la biodiversité posent problème. Plusieurs solutions ont été avancées pour concilier ces deux points de vue. Nous n'aborderons ici que celles faisant l'objet d'un large débat : la création de souches locales, débattue lors du colloque de février 1996 à Paris sur les « Introductions d'espèces dans les milieux aquatiques ", et l'introduction d'individus triploïdes.

La création de stocks locaux et l'entretien de ces géniteurs en captivité implique une gestion génétique rigoureuse de ces souches. En effet, la première étape consiste à analyser l'origine du pool génétique choisi pour être développé en pisciculture, mais il faut vérifier à plus ou moins longue échéance s'il n'existe pas de dérive génétique dans ces stocks, entraînant une perte de variabilité génétique et une certaine consanguinité. Ces problèmes peuvent apparaître 
même avec une gestion rigoureuse de la souche, et une participation régulière de gamètes issus de la nature est envisageable. CHEVASSUS (1989) a discuté des problèmes liés à la constitution de populations d'élevage destinées au repeuplement, à partir d'une ou plusieurs populations sources. II recense trois principaux facteurs maintenant une certaine variabilité : le nombre de reproducteurs utilisés, la proportion de chaque sexe dans le stock de reproducteurs et la contribution de chaque géniteur à la constitution de la descendance. Si l'élevage de cette souche locale s'effectue en effet selon le même protocole que pour la production de truites domestiques, la part des causes d'échec des repeuplements liée au déficit d'apprentissage sera maintenue.

Une autre proposition consiste à repeupler avec des truites triploïdes stériles, individus dont le génome est composé, par manipulation, de $3 n$ chromosomes (contre $2 n$ pour toute truite normalement constituée). Cette solution, déjà préconisée par GUYOMARD (1989 b), ne s'est pas développée avec le succès escompté, malgré son intérêt évident pour la conservation du génome des populations sauvages. Notons que plusieurs précautions doivent être prises. II faut d'abord contrôler le maintien de ces “ $3 n$ ” dans le milieu. D'autre part, ces truites ne pouvant se reproduire, ne pourront pas introgresser les populations sauvages, mais il convient de contrôler la compétition pour le territoire ou la nourriture qui peut s'instaurer avec les truites résidentes. Enfin, bien que stériles, les mâles « $3 n$ » ont un comportement de mâles sur les frayères et pourraient réduire la participation des mâles sauvages et faire baisser le taux de fécondation.

Enfin, d'aucuns pourraient prétendre à l'arrêt total des repeuplements et laisser le recrutement local ne se faire que par la reproduction naturelle. Cet arrêt pourrait se faire partout ou seulement dans des " sanctuaires génétiques", zones identifiées comme indemnes de toutes traces de repeuplement et génétiquement originales (GUYOMARD, 1989 b). Ces zones étaient identifiées uniquement sur des données électrophorétiques (KRIEG et GUYOMARD, 1983 ; nos données).

Les allozymes ont montré leur capacité à détecter l'influence des souches de pisciculture dans les populations naturelles méditerranéennes, même si l'information qu'ils donnent doit être confrontée à celle fournie par les autres marqueurs moléculaires. En revanche, il n'existe pas de locus allozymiques diagnostiques entre les souches d'élevage et les populations naturelles du versant atlantique, la différenciation entre ces deux taxons étant trop faible. Les microsatellites ont montré leur potentialité en région méditerranéenne. Ils seront employés prochainement pour distinguer la partie naturelle de la partie domestique des peuplements du versant atlantique. Ils permettront de décrire la biodiversité de ces régions et d'estimer l'impact des repeuplements et, par là même, leur efficacité.

\section{REMERCIEMENTS}

Les résultats décrits dans ce texte ont été obtenus grâce à plusieurs financements, qui ont donné lieu aux quatre rapports cités. Nous en remercions l'AAPPMA " La Gauloise " de Lodève (Hérault), l'ADESV (Association pour la Défense de l'Eau et des sites de la vallée de la Vis), le Club Halieutique Interdépartemental, la Fédération Départementale du Vaucluse, le Conseil Supérieur de la Pêche (convention 93-179), la Communauté Européenne (contrat EV5VCT920097), I'ONF services de l'Aude, l'association TFP (Truites Fario Pyrénéennes) (incluant les Fédérations Départementales de l'Ariège, l'Aude, la Haute Garonne, les Pyrénées Atlantiques, les Hautes Pyrénées et les Pyrénées Orientales), et le Parc Naturel Régional de Corse. Nous avons également bénéficié, lors des pêches, de l'appui logistique du Conseil Supérieur de la Pêche 8ème délégation, de la DIREN de Corse et des Fédérations Départementales déjà citées, ainsi que celles de l'Hérault et de Corse, qu'ils en soient remerciés, ainsi que les piscicultures de Brasac (Tarn) et de La Canourgue (Lozère). Enfin, une partie de ces études s'est faite en collaboration étroite avec l'Equipe Environnement Aquatique et Aquaculture de I'ENSA de Toulouse. Nous remercions également F. KRIEG pour ses suggestions judicieuses. 


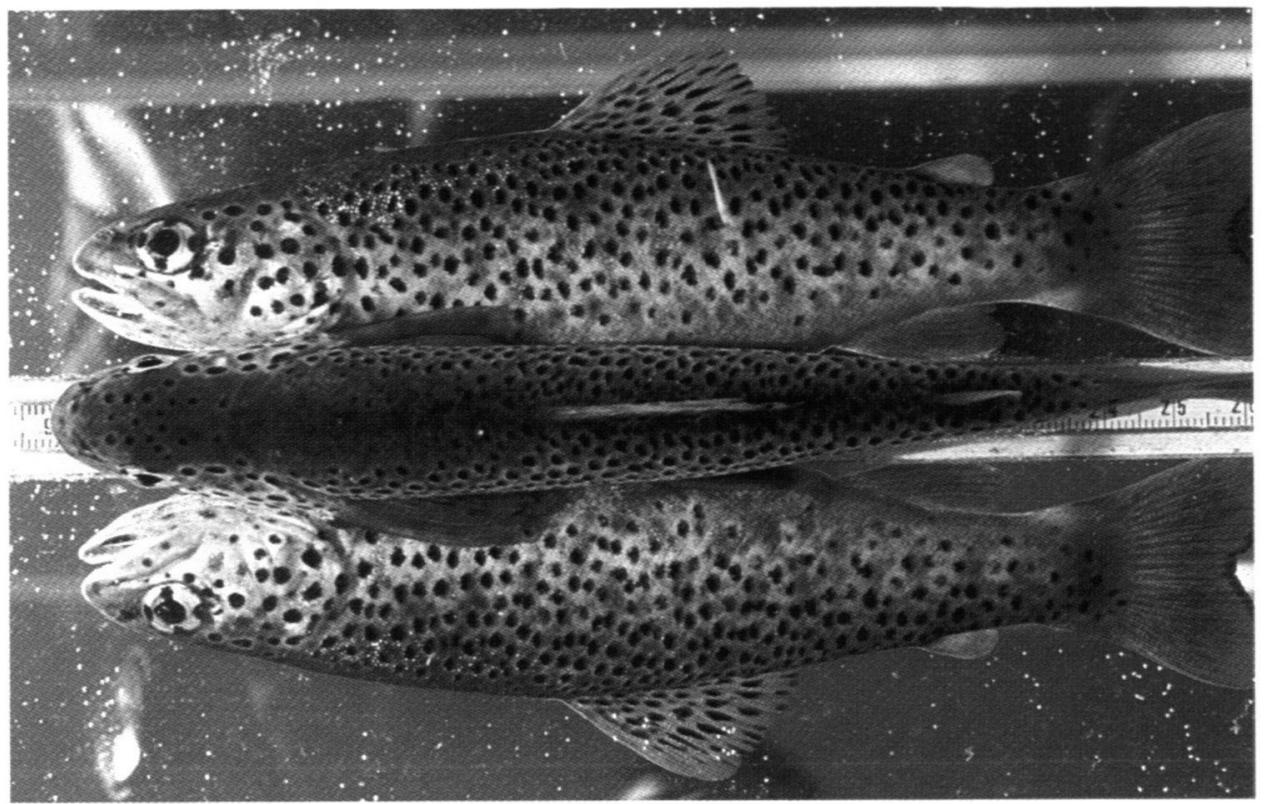

\section{Photo 1}

Morphotype méditerranéen (ici, une truite du Riu Ferrer, affluent du Tech, Pyrénées Orientales). Crédit photographique : Equipe Environnement Aquatique, ENSA Toulouse.

\section{Photo 1}

Mediterranean morphotype (here, an individual from Riu Ferrer river, a tributary of Tech river, Eastern Pyrenees).

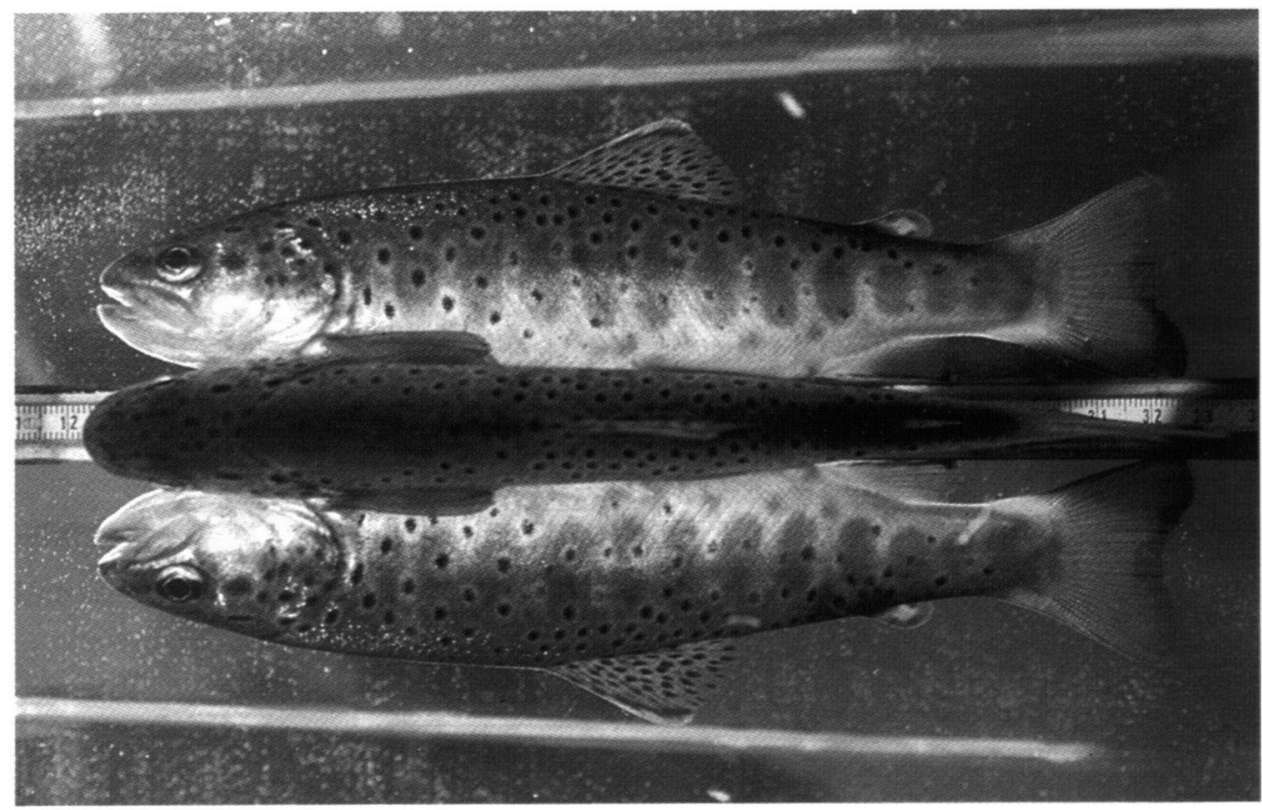

\section{Photo 2}

Morphotype atlantique domestique (ici, une truite capturée dans la Nohède, affluent de la Têt, Pyrénées Orientales). Crédit photographique : Equipe Environnement Aquatique, ENSA Toulouse.

\section{Photo 2}

Domestic morphotype belonging to the Atlantic group (here, an individual from Nohède river, a tributary of Têt river, Eastern Pyrenees). 


\section{BIBLIOGRAPHIE}

ALLENDORF F.W., MITCHELL N., RYMAN N., STAHL G., 1977. Isozyme loci in brown trout, Salmo trutta L. : detection and interpretation from population data. Hereditas, 86 , 179-190.

ALLENDORF F.W., UTTER F.M., 1979. Population genetics. In Fish Physiology, HOAR W.S., RANDALL D.J., BRETT J.R. (eds.), 407-454, New York, Academic Press.

ALLENDORF F.W., RYMAN N., UTTER F., 1987. Genetics and fisheries management : past, present and future. In Population genetics and fisheries fanagement, 1-19, RYMAN N., UTTER F. (eds.).

ALTUKHOV Y.P., 1981. The stock concept from the viewpoint of population genetics. Can. J. Fish. Aquat. Sci., 38, 1523-1538.

AURELLE D., 1996. Différenciation génétique chez la truite commune (Salmo trutta fario, L.) des Pyrénées-Atlantiques ; apport des microsatellites. D.E.A., Université Montpellier II, 29 p.

BARBAT-LETERRIER A., GUYOMARD R., KRIEG F., 1989. Introgression between introduced domesticated strains and Mediterranean native populations of brown trout (Salmo trutta L.). Aquatic living resources, 2, 215-223.

BEAUDOU D., 1993. Impact des déversements de truites domestiques dans les populations naturelles de truites communes, Salmo trutta fario. Etude dynamique et génétique. Cas du bassin de l'Orb, Hérault. Thèse de doctorat, Université de Montpellier II, 308 p.

BEAUDOU D., CATTANEO-BERREBI G., POTEAUX C., BERREBI P., 1993. Impact génétique de la réintroduction de truite commune (Salmo trutta fario) dans des populations du sud de la France. Bull. Soc. Zool. Fr., Evol. Zool., 118 (3), 311-312.

BEHNKE R.J., 1972. The systematics of salmonid fishes of recently glaciated lakes. J. Fish. Res. Bd. Canada, 29, 639-671.

BELKHIR K., BORSA P., GOUDET J., BONHOMME F., (soumis). Genetix 2.0, P.C. Gén. Sél. Evol.

BERNATCHEZ L., GUYOMARD R., BONHOMME F., 1992. DNA sequence variation of the mitochondrial control region among geographically and morphologically remote European brown trout Salmo trutta populations. Mol. Ecol., 1, 161-173.

BERREBI P., 1995 a. Etude génétique des truites fario de la Sorgue. Rapport final, février 1995, Université Montpellier II, $8 \mathrm{p}$.

BERREBI P., 1995 b. Analyse génétique des truites fario des rivières méditerranéennes des Pyrénées françaises; marqueurs enzymatiques. Rapport de synthèse, mars 1995, Université Montpellier II, $17 \mathrm{p}$.

BERREBI P., 1995 c. Etude génétique des truites de Corse. Rapport final, novembre 1995, Université Montpellier II, $36 \mathrm{p}$.

BERREBI P., 1995 d. Etude génétique des truites des affluents du Haut Hérault : Vis, Brèze, Laurounet, Lergue et Soulondre. Rapport technique, décembre 1995, Université Montpellier II, $7 \mathrm{p}$.

BUTH D.G., 1984. The application of electrophoretic data in systematic studies. Ann. Rev. Ecol. Syst., 15, 501-522.

CHEVASSUS B., 1989. Aspects génétiques de la constitution de populations d'élevage destinées au repeuplement. Bull. Fr. Pêche Piscic., 314 (3), 146-169.

DOBZHANSKY T., 1950. Genetics and the origin of species. New York, Columbia University Press.

ELLIOTT J.M., 1989. Wild brown trout Salmo trutta : an important national and international resource. Freshwater Biol., 21, 1-5. 
ESTOUP A., PRESA P., KRIEG F., VAIMAN D., GUYOMARD R., 1993. (CT) $n$ and (GT) $n$ microsatellites : a new class of genetic markers for Salmo trutta L., brown trout. Heredity, 71, 488-496.

FRASER J.M., 1981. Comparative survival and growth of planted wild, hybrid and domestic strains of brook trout (Salvelinus fontinalis) in Ontario lakes. Can. J. Fish. Aquat. Sci., 38, 1672-1684.

GARCIA DE LEANIZ C.E., VERSPOOR A., HAWKINS D., 1989. Genetic determination of the contribution of stocked and wild Atlantic salmon, Salmo salar L., to the angling fisheries in two Spanish rivers. J. Fish. Biol., 35 (suppl. A), 261-270.

GUYOMARD R., 1989 a. Diversité génétique de la truite commune. Bull. Fr. Pêche Piscic., 314 , 118-135.

GUYOMARD R., 1989 b. Gestion génétique des populations naturelles : l'exemple de la truite commune. Bull. Fr. Pêche Piscic., 314, 136-145.

GUYOMARD R., KRIEG F., 1983. Electrophoretic variation in six populations of brown trout (Salmo trutta L.). Can. J. Genet. Cytol., 25, 403-413.

GUYOMARD R., GREVISSE G., OURY F.X., DAVAINE P., 1984. Evolution de la variabilité génétique inter et intrapopulations de populations de salmonidés issus de mêmes pools géniques. Can. J. Fish. Aquat. Sci., 41, 1024-1029.

HAMILTON K.E., FERGUSON A., TAGGART J.B., TOMASSON T., WALKER A., FAHY E., 1989. Post-glacial colonisation of brown trout, Salmo trutta L. : Ldh-5 as a phylogeographic marker locus. J. Fish Biol., 35, 651-664.

HINDAR K., RYMAN N., UTTER F., 1991. Genetic effects of cultured fish on natural fish populations. Can. J. Fish. Aquat. Sci., 48, 945-957.

KRIEG F., GUYOMARD R., 1983. Mise en évidence électrophorétique d'une forte différenciation génétique entre populations de truite fario de Corse. C. R. Acad. Sci. Paris, 296, 1089-1094.

KRIEG F., GUYOMARD R., 1985. Population genetic of French brown trout, (Salmo trutta L.) : large geographical differenciation of wild populations and high similarity of domesticated stocks. Génét. Sél. Evol., 17 (2), 225-242.

LEARY R., ALLENDORF F.W., SAGE G., 1995. Hybridization and introgression between introduced and native fish. Am. Fish. Soc. Symp., 15, 91-101.

LETERRIER A., GUYOMARD R., KRIEG F., 1989. Introgression between introduced domesticated strains and Mediterranean native populations of brown trout, Salmo trutta L.. Aquat. Living Resour., 2, 215-223.

MACCRIMMON H.R., MARSHALL T.L., 1968. World distribution of brown trout, Salmo trutta. J. Fish Res. Board Can., 25, 25-27.

MAYR E., 1974. Populations, Espèces et Evolution. Paris, Hermann.

MORAN P., PENDAS A.M., GARCIA-VAZQUEZ E., IZQUIERDO J., 1991. Failure of a stocking policy, of hatchery reared brown trout, Salmo trutta L., in Asturias, Spain, detected using $\mathrm{LDH}-5^{*}$ as a genetic marker. J. Fish Biol., 39 (suppl. A), 117-121.

MORAN P., GARCIA-VASQUEZ E., PENDAS A.M., IZQUIERDO J.I., MARTIN VENTURA J.A., 1993. Status of genetic conservation in salmonid populations from Asturian rivers, north of Spain. In Genetic Conservation of Salmonid Fishes, CLOUD J.G., THORGAARD G.H. (eds.), NATO ASI Series : Life Sciences 248, New York, Plenum Press.

PASTEUR N., PASTEUR G., BONHOMME F., CATALAN J., BRITTON-DAVIDIAN J., 1987. Manuel de génétique par électrophorèse des protéines. Paris, Lavoisier. 
POTEAUX C., 1992. Devenir des alevins de truite commune (Salmo trutta) déversés expérimentalement dans le Tes (affluent de l'Orb). Etude dynamique et génétique. DEA Université Montpellier II, $27 \mathrm{p}$.

POTEAUX C., 1995. Analyse des interactions génétiques entre formes sauvages et domestiques chez la truite commune, Salmo trutta fario. Thèse de doctorat, Université de Montpellier II, $110 \mathrm{p}$.

POTEAUX C., BONHOMME F., BERREBI P., (soumis). Microsatellite polymorphism and genetic impact of restocking in Mediterranean brown trout (Salmo trutta fario L.).

PRESA P., KRIEG F., ESTOUP A., GUYOMARD R., 1994. Diversité et gestion génétique de la truite commune : apport de l'étude du polymorphisme des locus protéiques et microsatellites. Génét. Sél. Evol., 26, 183-202.

RYMAN N., ALLENDORF F.W., STAHL G., 1979. Reproductive isolation with little genetic divergence in sympatric populations of brown trout (Salmo trutta). Genetics, 92, 247-262.

RYMAN N., UTTER F., ALLENDORF F.W., BUSACK C., SHAKLEE J., 1993. Genetic concern about hatchery populations - a comment on the conclusions of Nyman and Ring. J. Fish Biol., 42, 471-480.

SASTRE C., BENTATA V., 1997. Proposition de définitions pour les termes biogéographiques utilisés. Bull. Fr. Pêche Piscic., 344-345.

SHAKLEE J.B., ALLENDORF F.W., MORIZOT D.C., WHITT G.S., 1990. Gene nomenclature for protein-coding loci in fish. Trans. Am. Fish. Soc., 119, 2-15.

UTTER F.M., SEEB J.E., 1990. Genetic marking of fishes : overview focusing on protein variation. Am. Fish. Soc. Symp., 7, 426-438.

WAPLES R.S., 1991. Genetic interactions between hatchery and wild salmonids : Lessons from the Pacific Northwest. Can. J. Fish. Aquat. Sci., 48 (Suppl.1), 124-131.

WEIR B.S., COCKERHAM C.C., 1984. Estimating F-statistics for the analysis of population structure. Evolution, 38, 1358-1370. 